Internat. J. Math. \& Math. Sci.

Vol. 24, No. 12 (2000) 851-855

S0161171200003471

(C) Hindawi Publishing Corp.

\title{
CERTAIN REMARKS ON A CLASS OF EVOLUTION QUASI-VARIATIONAL INEQUALITIES
}

\author{
A. H. SIDDIQI and PAMMY MANCHANDA
}

(Received 17 May 1999 and in revised form 9 March 2000)

\begin{abstract}
We prove two existence theorems, one for evolution quasi-variational inequalities and the other for a time-dependent quasi-variational inequality modeling the quasistatic problem of elastoplasticity with combined kinetic-isotropic hardening.
\end{abstract}

Keywords and phrases. Evolution quasi-variational inequalities, coercive, weakly convergent, weakly continuous, weak topology.

2000 Mathematics Subject Classification. Primary 49J40, 47A63, 65K10.

1. Introduction. A comprehensive updated account of the elliptic quasi- and evolution quasi-variational inequalities is presented by Baiocchi and Capelo [1]. Evolution variational inequalities have been investigated by Lions and Stampacchia [9], Brézis [2, 3], Duvaut and Lions [4], and Lions [8]. A related general problem of Moreau's sweeping process can be found in [10]. Elliptic variational and quasi-variational inequalities are discussed in [1] along with the references on evolution variational inequalities. In 1994, Mosco [11] formulated the implicit Signorini problem in terms of the elliptic quasi-variational inequality problem and indicated many open problems including the numerical methods and the probable outlines of the method. Recently, Kočvara and Outrata [6] and Outrata and Zowe [12] have studied numerical solutions of quasivariational inequalities using techniques of nonsmooth optimization. The evolution quasi-variational inequality modeling the evolving shape of a growing pile has been studied by Prigozhin [13]. Han, Reddy, and Schroeder [5] have investigated a new class of evolution inequalities where rate quantities occur in all of its terms. An inequality of this type represents, for example, the quasi-static evolution of an elastoplastic body where the stress law is of the linear kinematic or isotropic hardening type. Such a variational inequality may be useful in the modeling of financial derivatives and option pricing, see Wilmott et al. [14]. In this paper, we discuss the existence of solutions of the implicit evolution quasi-variational inequalities. In Section 2, we present an existence theorem which is closely related to an open problem mentioned in [13]. The evolution quasi-variational inequality problem of the Han-Reddy-Schroeder type is considered in Section 3.

2. Evolution quasi-variational inequalities of Bensoussan-Lions-Mosco type. Let $H$ be a Hilbert space with the inner product $\langle, \cdot$,$\rangle and the induced norm \|\cdot\|=$ $(\langle\cdot, \cdot\rangle)^{1 / 2}$. Let $L_{2}(0, T ; H)$ denote the space of all measurable functions $u:(0, T) \rightarrow H$, then it is a Hilbert space with respect to the inner product 


$$
\langle u, v\rangle_{L_{2}(0, T, H)}=\int_{0}^{T}\langle u(t), v(t)\rangle_{H} d t
$$

If $H^{*}$ is the topological dual of $H$, then the dual of $L_{2}(0, T, H)=L_{2}\left(0, T, H^{*}\right)$ in case of real $H$.

Let $H^{1, p}(0, T, H)$ or, very often, $H^{p}(0, T, H)$ denote the space of functions $f \in$ $L_{p}(0, T, H)$ such that their distributional derivatives $D_{f}$ also belong to $L_{p}(0, T, H)$. $H^{p}(0, T, H)$ equipped with the norm

$$
\|f\|_{H^{P}(0, T, H)}^{2}=\|f\|_{L_{p}(0, T, H)}^{2}+\left\|D_{f}\right\|_{L_{p}(0, T, H)}^{2}
$$

is a Banach space and $H^{2}(0, T, H)$ is a Hilbert space. Let $F \in H^{2}\left(0, T, H^{*}\right)$ and let $C$ be a nonempty, closed, and convex subset of $H^{2}(0, T, H)$. Further, let $a(\cdot, \cdot)$ be a symmetric bounded and coercive bilinear form. We consider here the following problem.

BenSOUSSAN-Lions-Mosco PROBLem. Find $u \in K(u) \cap C$ such that

$$
\begin{aligned}
\left\langle\frac{\partial u}{\partial t}, v-u(t)\right\rangle+a(u, v-u(t)) & \geq\langle F(t), v-u(t)\rangle \\
\forall v & \in K(u), u(t) \in K, u(0)=u^{0} \in K\left(u^{0}\right) .
\end{aligned}
$$

Here, $K(\cdot): C \rightarrow 2^{H}$ is a set-valued mapping defined on $C$ which associates a nonempty closed convex subset $K(z)$ of $H$ with every $z \in C$; and $F(t) \in H^{*}$.

We also assume the following conditions.

The set-valued map $z \rightarrow K(z)$ is weakly continuous on $C$, in the sense that for every sequence $z_{h} \in C$ converging to some $z \in C$, weakly in $H$, the sequence of subsets $K\left(z_{h}\right)$ converges to $K(z)$ in $H$, equivalently to the following conditions:

(i) if $w_{h} \in K\left(z_{h}\right)$ and $w_{h}$ converges weakly to some $w$ in $H$, then

$$
w \in K(z)
$$

(ii) for every $v \in K(z)$, there exists $v_{h} \in K\left(z_{h}\right)$ such that $v_{h}$ converges strongly to $v$ in $H$.

We further assume that sets $K(z)$ for all $z \in C$ have a nonempty intersection, that is, $u^{0} \in \bigcap_{h \in H} K(h)$ and $S$ maps $C$ into itself.

THEOREM 2.1. Let $a(\cdot, \cdot)$ be a coercive continuous bilinear form. Then, under the above conditions, (2.3) has at least one solution.

Proof OF TheOrem 2.1. We can associate with problem (2.3) the following family of variational inequalities indexed by the fixed $z \in C$, namely $u \in K(z)$,

$$
\begin{aligned}
\left\langle\frac{\partial u}{\partial t}, v-u(t)\right\rangle+a(u, v-u(t)) & \geq\langle F(t), v-u(t)\rangle \\
\forall v & \in K(z), u(t) \in K(z), u(0)=u^{0} \in K(z) .
\end{aligned}
$$

Equation (2.5) can be written in the form:

for some $u \in K(z)$

$$
\left\langle\frac{\partial u}{\partial t}+A u(t)-F(t), v-u(t)\right\rangle \geq 0
$$


where $A$ is a coercive continuous linear operator induced by $a(\cdot, \cdot)$. By the well-known existence theorem of the evolution variational inequality (cf. [2, 3, 4, 8, 9]) for each fixed $z \in C$, (2.6) has a unique solution $w$, which we denote by $w=S(z)$. This defines the map

$$
S: C \rightarrow H, \quad w=S(z)
$$

It is clear that (2.7) is the selection map associated with (2.3) and the existence of its solution is equivalent to the fixed point problem

$$
u \in C: S(u)=u \text {. }
$$

The existence of a fixed point is guaranteed by the Schauder-Tychonov fixed point theorem if we show that $S(C)$ is bounded and $S$ is a weakly continuous map with respect to the weak topology of $H$. By the hypothesis of the theorem, we find that every $w=S(z), w \in C$, satisfies

$$
\left\|\frac{\partial w}{\partial t}\right\| \geq \mu\left\|w^{*}-w\right\|^{2}
$$

where $v$ in (2.6) is replaced by $w^{*}$. This shows that $S(C)$ is bounded.

We now prove that $S: C \rightarrow C$ is continuous for the weak topology of $H$. Let $z_{h}$ be a sequence in $C$, converging weakly to $z$ and let $w_{h} \in K\left(z_{h}\right)$. Since $\left\|w_{h}\right\|$ is bounded, there is a subsequence, say $w_{h}$, which converges weakly to some $w$. By (i), $w \in K(z)$. Furthermore, by (ii), for every $v \in K(z)$, there exists $v_{h} \in K\left(z_{h}\right)$ such that $v_{h}$ converges to $v$ strongly.

Replacing $u$ by $v_{h}$ in (2.6) and taking the limit as $h \rightarrow \infty$, we get

$$
\left\langle\frac{\partial w}{\partial t}+A w(t)-F(t), v-w(t)\right\rangle \geq 0
$$

Therefore $w=S(z)$. By the uniqueness of solution of (2.6), $w_{h}$ converges weakly to $w$. By restricting $S$ to the set $C^{*}=C \cap\{z:\|z\| \leq r\}, r \gg 0$, which is a weak compact subset of $H$, we see that $S$ maps $C^{*}$ into itself provided $r$ is sufficiently large. This proves the existence of a fixed point of $S$ and consequently a solution of (2.3).

REMARK 2.2. Very recently our attention has been drawn to a preprint of December 1997 by Kunze (University of Köln) and Monteiro Marques (University of Lisbon) entitled "On parabolic quasi-variational inequalities and state-dependent sweeping process" where a result similar to Theorem 2.1 has been proved by a different method. This paper has now appeared [7]. In a recent paper of Lions [8], it is observed in Remark 6.3 that one can extend the methods of the present paper to some quasivariational inequalities.

It may be observed that the stationary case models the implicit Signorini problem

$$
\begin{aligned}
& -\Delta u+u=\varphi \quad \text { in } \Omega, \\
& u-\left(h-\int_{\Gamma} \varphi \frac{\partial u}{\partial n}\right) \geq 0, \quad \frac{\partial u}{\partial n} \geq 0, \\
& {\left[u-\left(h-\int_{\Gamma} \varphi \frac{\partial u}{\partial n}\right)\right] \frac{\partial u}{\partial n}=0 \quad \text { on } \partial \Omega .}
\end{aligned}
$$


Many important physical phenomena can be interpreted in this form.

The number of solutions of (2.3) and its numerical analysis like error estimation, stability and convergence are open questions even in the stationary case. Mosco [11, page 29] has made certain suggestions in this connection. Kočvara and Outrata [6] and Outrata and Zowe [12] have studied the existence and numerical solutions of certain elliptic quasi-variational inequalities. Numerical solutions of (2.3), in some special cases, have been considered by Prigozhin [13].

3. Evolution quasi-variational inequality of Han-Reddy-Schroeder type. Han, Reddy, and Schroeder [5] have studied the following evolution variational inequality problem and its application to elastoplasticity:

Find $u:[0, T] \rightarrow H, u(0)=0$ such that for almost all $t \in[0, T], \dot{u}(t) \in K$ and

$$
a(u(t), v-\dot{u}(t))+j(\dot{u}(t)) \geq\langle F(t), v-\dot{u}(t)\rangle, \quad \forall v \in K .
$$

We prove here an existence theorem for the following evolution quasi-variational inequality problem:

Find $u \in K(u) \cap C, u(0)=0$ such that for almost all $t \in[0, T]$,

$$
a(u(t), v-\dot{u}(t)) \geq\langle F(t), v-\dot{u}(t)\rangle, \quad \forall v \in K(u) .
$$

THEOREM 3.1. Under the hypothesis of Theorem 2.1, inequality (3.2) has at least one solution.

Proof OF Theorem 3.1. The problem of evolution quasi-variational inequality can be expressed as the following family of variational inequalities indexed by the vector $z \in C$ :

$$
w \in K(z), \quad a(w, v-\dot{w}(t)) \geq\langle F(t), v-\dot{w}(t)\rangle, \quad \text { for every } v \in K(z) .
$$

By [5, Theorem 4.3], (3.2) has a unique solution $w$, denoted by $w=S(z)$ which defines the map

$$
S: C \rightarrow C, \quad w=S(z)
$$

that is, $\dot{z}(t)$ is associated with every $z$ and is itself mapped to $P_{K} \dot{z}(t)-\rho(A z-F(t))$, where $A$ is the bounded linear operator induced by $a(\cdot, \cdot)$, namely

$$
\langle A z, v\rangle=a(z, v) .
$$

By the arguments used in the proof of Theorem 2.1, it can be proved that $S$ has a fixed point which proves the existence of a solution of (3.2).

It may be observed that the uniqueness, stability and finite element analysis of this class of evolution variational equalities are open problems.

ACKNOWLEDGEMENT. Siddiqi would like to express his gratitude to King Fahd University of Petroleum and Minerals, Dhahran, Saudi Arabia, for providing excellent research facilities. The authors are thankful to the referee for valuable suggestions. 


\section{REFERENCES}

[1] C. Baiocchi and A. Capelo, Variational and Quasivariational Inequalities, Applications to free boundary problems. Translated from the Italian by Lakshmi Jayakar. A WileyInterscience Publication, John Wiley \& Sons Inc., New York, 1984. MR 86e:49018. Zbl 551.49007.

[2] H. Brézis, Équations et inéquations non linéaires dans les espaces vectoriels en dualité, Ann. Inst. Fourier (Grenoble) 18 (1968), no. 1, 115-175 (French). MR $42 \# 5113$. Zbl 169.18602.

[3] _ Problèmes unilatéraux, J. Math. Pures Appl. (9) 51 (1972), 1-168 (French). MR 55\#1166. Zbl 237.35001.

[4] G. Duvaut and J.-L. Lions, Inequalities in Mechanics and Physics, Translated from the French by C. W. John. Grundlehren der Mathematischen Wissenschaften, vol. 219, Springer-Verlag, Berlin, Heidelberg, New York, 1976. MR 58\#25191. Zbl 331.35002.

[5] W. Han, B. D. Reddy, and G. C. Schroeder, Qualitative and numerical analysis of quasistatic problems in elastoplasticity, SIAM J. Numer. Anal. 34 (1997), no. 1, 143-177. MR 98a:73029. Zbl 933.74010.

[6] M. Kočvara and J. V. Outrata, On a class of quasi-variational inequalities, Optim. Methods Softw. 5 (1995), 275-295.

[7] M. Kunze and M. D. P. Monteiro Marques, On parabolic quasi-variational inequalities and state-dependent sweeping processes, Topol. Methods Nonlinear Anal. 12 (1998), no. 1, 179-191. MR 2000b:34102. Zbl 923.34018.

[8] J.-L. Lions, Parallel algorithms for the solution of variational inequalities, Interfaces Free Bound. 1 (1999), no. 1, 3-16. Zbl 941.65063.

[9] J.-L. Lions and G. Stampacchia, Variational inequalities, Comm. Pure Appl. Math. 20 (1967), 493-519. MR 35\#7178. Zbl 152.34601.

[10] M. D. P. Monteiro Marques, Differential Inclusions in Nonsmooth Mechanical Problems, Shocks and dry friction. Progress in Nonlinear Differential Equations and their Applications, vol. 9, Birkhäuser Verlag, Basel, 1993. MR 94g:34003. Zbl 802.73003.

[11] U. Mosco, Some introductory remarks on implicit variational problems, Recent Developments in Applicable Mathematics (A. H. Siddiqi, ed.), Macmillan India Limited, 1994, pp. 1-45.

[12] J. V. Outrata and J. Zowe, A Newton method for a class of quasi-variational inequalities, Comput. Optim. Appl. 4 (1995), no. 1, 5-21. MR 95k:49023. Zbl 827.49007.

[13] L. Prigozhin, Variational model of sandpile growth, European J. Appl. Math. 7 (1996), no. 3, 225-235. MR 97j:73065. Zbl 913.73079.

[14] P. Wilmott, J. Dewynne, and S. Howison, Option Pricing-Mathematical Models and Computation, Oxford Finance Press, 1994.

A. H. SidDiQI: DePARTMENT OF MATHEMATICAl SCIENCES, King FAHD UniVERSity OF PETROLEUM AND MiNERALS, DHAHRAN 31261, SAUDI ARABIA

E-mail address: ahasan@kfupm.edu.sa

Pammy Manchanda: Department of Mathematics, Gurunanak DeV University, AMRITSAR-143005, INDIA

E-mail address: kmanch@j 1a.vsn1.net. in 


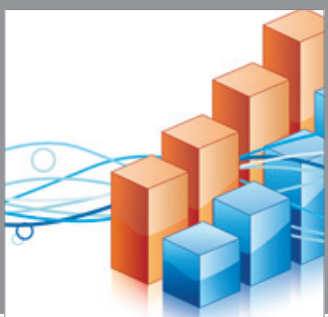

Advances in

Operations Research

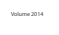

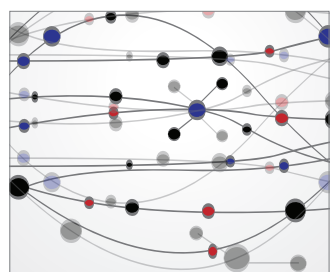

\section{The Scientific} World Journal
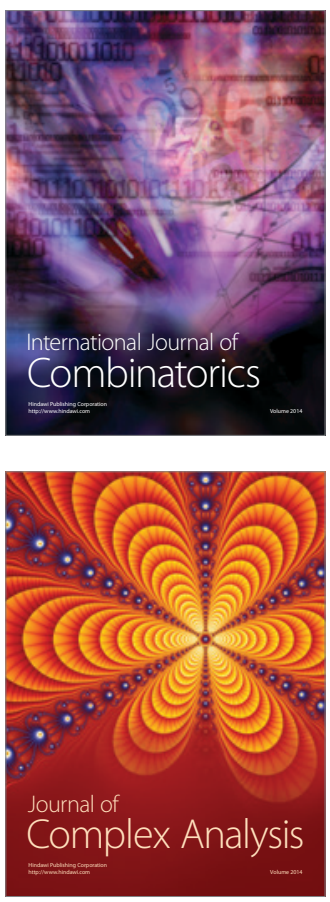

International Journal of

Mathematics and

Mathematical

Sciences
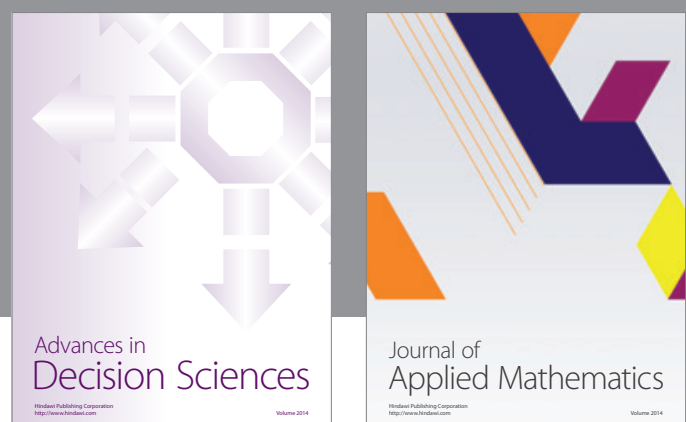

Journal of

Applied Mathematics
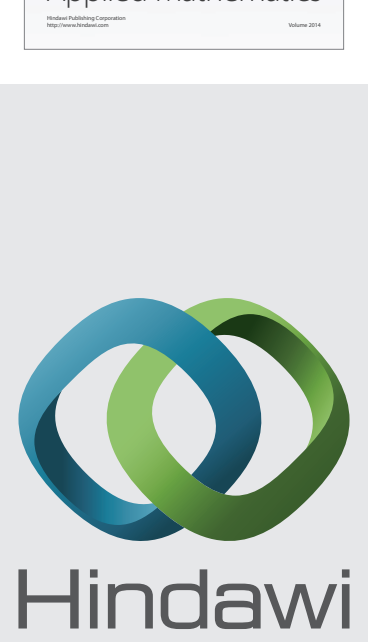

Submit your manuscripts at http://www.hindawi.com
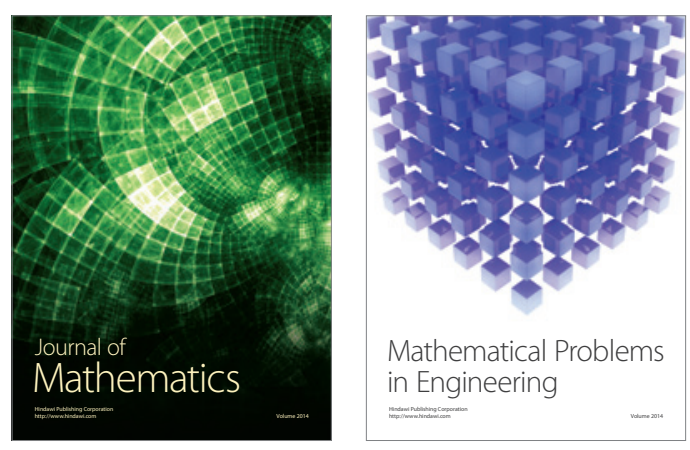

Mathematical Problems in Engineering
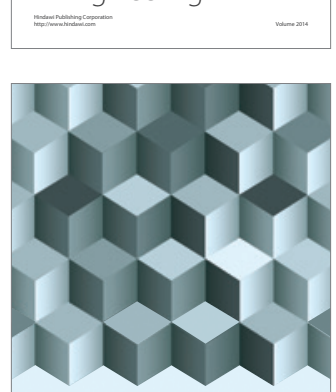

Journal of

Function Spaces
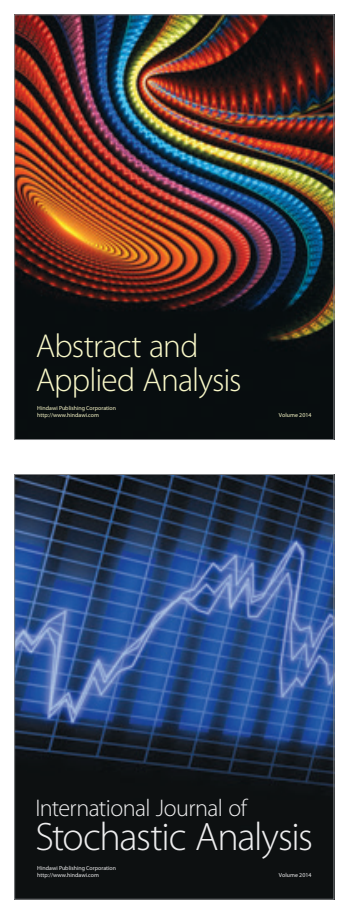

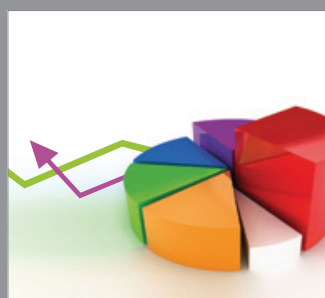

ournal of

Probability and Statistics

Promensencen
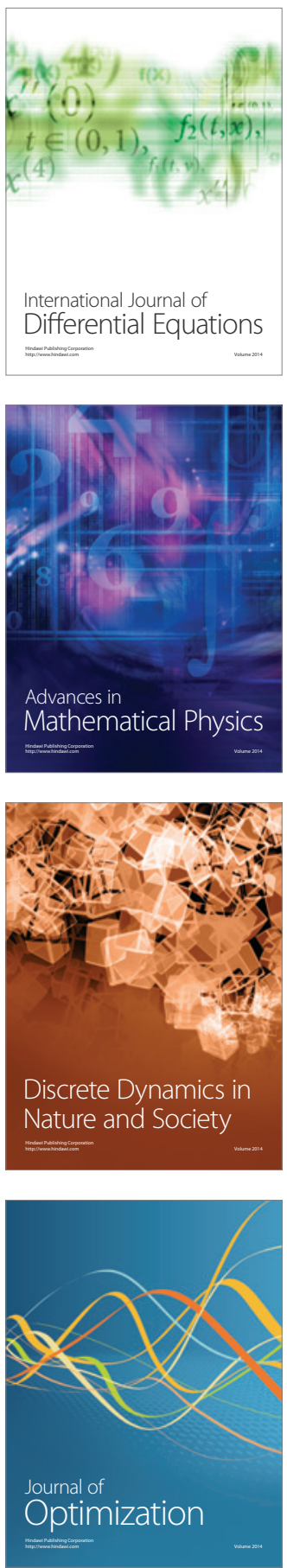